\title{
Shrinking Projection Method for Fixed Point Problems of an Infinite Family of Strictly Pseudocontractive Mappings and the System of Cocoercive Quasivariational Inclusions Problems in Hilbert Spaces
}

\author{
Pattanapong Tianchai \\ Faculty of Science, Maejo University, Chiangmai 50290, Thailand \\ Correspondence should be addressed to Pattanapong Tianchai, pattana@mju.ac.th \\ Received 12 April 2011; Accepted 3 May 2011 \\ Academic Editor: C. Zhu \\ Copyright (C) 2011 Pattanapong Tianchai. This is an open access article distributed under the \\ Creative Commons Attribution License, which permits unrestricted use, distribution, and \\ reproduction in any medium, provided the original work is properly cited. \\ This paper is concerned with a common element of the set of common fixed points for an infinite \\ family of strictly pseudocontractive mappings and the set of solutions of a system of cocoercive \\ quasivariational inclusions problems in Hilbert spaces. The strong convergence theorem for the \\ above two sets is obtained by a general iterative scheme based on the shrinking projection method, \\ and the applicability of the results is shown to extend and improve some well-known results \\ existing in the current literature.
}

\section{Introduction}

Throughout this paper, we always assume that $C$ is a nonempty closed convex subset of a real Hilbert space $H$ with inner product and norm denoted by $\langle\cdot, \cdot\rangle$ and $\|\cdot\|$, respectively, $2^{H}$ denoting the family of all the nonempty subsets of $H$.

Let $B: H \rightarrow H$ be a single-valued nonlinear mapping and $M: H \rightarrow 2^{H}$ a set-valued mapping. We consider the following quasivariational inclusion problem, which is to find a point $x \in H$,

$$
\theta \in B x+M x
$$

where $\theta$ is the zero vector in $H$. The set of solutions of problem (1.1) is denoted by $\mathrm{VI}(H, B, M)$. 
Recall that $P_{C}$ is the metric projection of $H$ onto $C$; that is, for each $x \in H$, there exists the unique point in $P_{C} x \in C$ such that $\left\|x-P_{C} x\right\|=\min _{y \in C}\|x-y\|$. A mapping $T: C \rightarrow C$ is called nonexpansive if $\|T x-T y\| \leq\|x-y\|$ for all $x, y \in C$. A point $x \in C$ is a fixed point of $T$ provided $T x=x$. We denote by $F(T)$ the set of fixed points of $T$; that is, $F(T)=\{x \in C: T x=x\}$. If $C$ is nonempty bounded closed convex subset of $H$ and $T$ is a nonexpansive mapping of $C$ into itself, then $F(T)$ is nonempty (see [1]). Recall that a mapping $A: C \rightarrow C$ is said to be

(i) monotone if

$$
\langle A x-A y, x-y\rangle \geq 0, \quad \forall x, y \in C
$$

(ii) $k$-Lipschitz continuous if there exists a constant $k>0$ such that

$$
\|A x-A y\| \leq k\|x-y\|, \quad \forall x, y \in C,
$$

if $k=1$, then $A$ is a nonexpansive,

(iii) pseudocontractive if

$$
\|A x-A y\|^{2} \leq\|x-y\|^{2}+\|(I-A) x-(I-A) y\|^{2}, \quad \forall x, y \in C,
$$

(iv) $k$-strictly pseudocontractive if there exists a constant $k \in[0,1)$ such that

$$
\|A x-A y\|^{2} \leq\|x-y\|^{2}+k\|(I-A) x-(I-A) y\|^{2}, \quad \forall x, y \in C,
$$

it is obvious that $A$ is a nonexpansive if and only if $A$ is 0 -strictly pseudocontractive,

(v) $\alpha$-strongly monotone if there exists a constant $\alpha>0$ such that

$$
\langle A x-A y, x-y\rangle \geq \alpha\|x-y\|^{2}, \quad \forall x, y \in C,
$$

(vi) $\alpha$-inverse-strongly monotone (or $\alpha$-cocoercive) if there exists a constant $\alpha>0$ such that

$$
\langle A x-A y, x-y\rangle \geq \alpha\|A x-A y\|^{2}, \quad \forall x, y \in C,
$$

if $\alpha=1$, then $A$ is said to be firmly nonexpansive; it is obvious that any $\alpha$-inversestrongly monotone mapping $A$ is monotone and $(1 / \alpha)$-Lipschitz continuous.

The existence of common fixed points for a finite family of nonexpansive mappings has been considered by many authors (see [2-5] and the references therein). 
In this paper, we study the mapping $W_{n}$ defined by

$$
\begin{gathered}
U_{n, n+1}=I, \\
U_{n, n}=\mu_{n} S_{n} U_{n, n+1}+\left(1-\mu_{n}\right) I, \\
U_{n, n-1}=\mu_{n-1} S_{n-1} U_{n, n}+\left(1-\mu_{n-1}\right) I, \\
\vdots \\
U_{n, k}=\mu_{k} S_{k} U_{n, k+1}+\left(1-\mu_{k}\right) I, \\
U_{n, k-1}=\mu_{k-1} S_{k-1} U_{n, k}+\left(1-\mu_{k-1}\right) I, \\
\vdots \\
U_{n, 2}=\mu_{2} S_{2} U_{n, 3}+\left(1-\mu_{2}\right) I, \\
W_{n}=U_{n, 1}=\mu_{1} S_{1} U_{n, 2}+\left(1-\mu_{1}\right) I,
\end{gathered}
$$

where $\left\{\mu_{i}\right\}$ is nonnegative real sequence in $(0,1)$, for all $i \in \mathbb{N}, S_{1}, S_{2}, \ldots$ from a family of infinitely nonexpansive mappings of $C$ into itself. It is obvious that $W_{n}$ is a nonexpansive mapping of $C$ into itself; such a mapping $W_{n}$ is called a $W$-mapping generated by $S_{1}, S_{2}, \ldots, S_{n}$ and $\mu_{1}, \mu_{2}, \ldots, \mu_{n}$.

Definition 1.1 (see [6]). Let $M: H \rightarrow 2^{H}$ be a multivalued maximal monotone mapping. Then, the single-valued mapping $J_{M, \lambda}: H \rightarrow H$ defined by $J_{M, \lambda}(u)=(I+\lambda M)^{-1}(u)$, for all $u \in H$, is called the resolvent operator associated with $M$, where $\lambda$ is any positive number and $I$ is the identity mapping.

Recently, Zhang et al. [6] considered the problem (1.1) and the problem of a fixed point of nonexpansive mapping. To be more precise, they proved the following theorem.

Theorem ZLC. Let $H$ be a real Hilbert space, $B: H \rightarrow H$ an $\alpha$-inverse-strongly monotone mapping, $M: H \rightarrow 2^{H}$ a maximal monotone mapping, and $T: H \rightarrow H$ a nonexpansive mapping. Suppose that the set $F(T) \cap \operatorname{VI}(H, B, M) \neq \emptyset$, where $\operatorname{VI}(H, B, M)$ is the set of solutions of quasivariational inclusion (1.1). Suppose that $x_{1}=x \in H$ and $\left\{x_{n}\right\}$ is the sequence defined by

$$
\begin{gathered}
y_{n}=J_{M, \lambda}\left(x_{n}-\lambda B x_{n}\right), \\
x_{n+1}=\alpha_{n} x+\left(1-\alpha_{n}\right) T y_{n},
\end{gathered}
$$

for all $n \in \mathbb{N}$, where $\lambda \in(0,2 \alpha)$ and $\left\{\alpha_{n}\right\} \subset(0,1)$ satisfying the following conditions:

(C1) $\lim _{n \rightarrow \infty} \alpha_{n}=0$ and $\sum_{n=1}^{\infty} \alpha_{n}=\infty$,

(C2) $\sum_{n=1}^{\infty}\left|\alpha_{n+1}-\alpha_{n}\right|<\infty$.

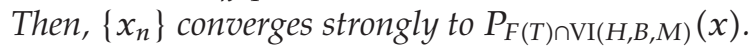

Nakajo and Takahashi [7] introduced an iterative scheme for finding a fixed point of a nonexpansive mapping by a hybrid method which is called that shrinking projection method (or CQ method) as in the following theorem. 
Theorem NT. Let $C$ be a nonempty closed convex subset of a real Hilbert space $H$. Let $T$ be a nonexpansive mapping of $C$ into itself such that $F(T) \neq \emptyset$. Suppose that $x_{1}=x \in C$ and $\left\{x_{n}\right\}$ is the sequence defined by

$$
\begin{gathered}
y_{n}=\alpha_{n} x_{n}+\left(1-\alpha_{n}\right) T x_{n}, \\
C_{n}=\left\{z \in C:\left\|y_{n}-z\right\| \leq\left\|x_{n}-z\right\|\right\}, \\
Q_{n}=\left\{z \in C:\left\langle x_{n}-z, x_{1}-x_{n}\right\rangle \geq 0\right\}, \\
x_{n+1}=P_{C_{n} \cap Q_{n}}\left(x_{1}\right), \quad \forall n \in \mathbb{N},
\end{gathered}
$$

where $0 \leq \alpha_{n} \leq \alpha<1$. Then, $\left\{x_{n}\right\}$ converges strongly to $P_{F(T)}\left(x_{1}\right)$.

In the same way, Kikkawa and Takahashi [8] introduced an iterative scheme for finding a common fixed point of an infinite family of nonexpansive mappings as follows:

$$
\begin{gathered}
y_{n}=W_{n} x_{n}, \\
C_{n}=\left\{z \in C:\left\|y_{n}-z\right\| \leq\left\|x_{n}-z\right\|\right\}, \\
Q_{n}=\left\{z \in C:\left\langle x_{n}-z, x_{1}-x_{n}\right\rangle \geq 0\right\}, \\
x_{n+1}=P_{C_{n} \cap Q_{n}}\left(x_{1}\right), \quad \forall n \in \mathbb{N},
\end{gathered}
$$

where $x_{1}=x \in C$ and $W_{n}$ is a $W$-mapping of $C$ into itself generated by $\left\{T_{n}: C \rightarrow C\right\}$ and $\left\{\mu_{n}\right\}$. They prove that, if $\Omega=\bigcap_{n=1}^{\infty} F\left(T_{n}\right) \neq \emptyset$, then the sequence $\left\{x_{n}\right\}$ generated by (1.11) converges strongly to $P_{\Omega}\left(x_{1}\right)$.

Recently, Su and Qin [9] modified the shrinking projection method for finding a fixed point of a nonexpansive mapping, for which the convergence rate of the iterative scheme is faster than that of the iterative scheme of Nakajo and Takahashi [7] as follows:

$$
\begin{gathered}
y_{n}=\alpha_{n} x_{n}+\left(1-\alpha_{n}\right) T x_{n}, \\
C_{n}=\left\{z \in C_{n-1} \cap Q_{n-1}:\left\|y_{n}-z\right\| \leq\left\|x_{n}-z\right\|\right\}, \quad n \geq 1, \\
Q_{n}=\left\{z \in C_{n-1} \cap Q_{n-1}:\left\langle x_{n}-z, x_{0}-x_{n}\right\rangle \geq 0\right\}, \quad n \geq 1, \\
C_{0}=\left\{z \in C:\left\|y_{0}-z\right\| \leq\left\|x_{0}-z\right\|\right\}, \\
Q_{0}=C, \\
x_{n+1}=P_{C_{n} \cap Q_{n}}\left(x_{0}\right), \quad \forall n \in \mathbb{N} \cup\{0\},
\end{gathered}
$$

where $x_{0}=x \in C$ and $T$ is a nonexpansive mapping of $C$ into itself. They prove that, under the parameter $0 \leq \alpha_{n} \leq \alpha<1$, if $F(T) \neq \emptyset$, then the sequence $\left\{x_{n}\right\}$ generated by (1.12) converges strongly to $P_{F(T)}\left(x_{0}\right)$. 
On the other hand, Tada and Takahashi [10] introduced an iterative scheme for finding a common element of the set of solutions of an equilibrium problem and the set of solutions of a fixed point problem of a nonexpansive mapping as follows:

$$
\begin{gathered}
u_{n} \in C \text { such that } F\left(u_{n}, y\right)+\frac{1}{r_{n}}\left\langle y-u_{n}, u_{n}-x_{n}\right\rangle \geq 0, \quad \forall y \in C, \\
y_{n}=\left(1-\alpha_{n}\right) x_{n}+\alpha_{n} T u_{n}, \\
C_{n}=\left\{z \in H:\left\|y_{n}-z\right\| \leq\left\|x_{n}-z\right\|\right\}, \\
Q_{n}=\left\{z \in H:\left\langle x_{n}-z, x_{1}-x_{n}\right\rangle \geq 0\right\}, \\
x_{n+1}=P_{C_{n} \cap Q_{n}}\left(x_{1}\right), \quad \forall n \in \mathbb{N},
\end{gathered}
$$

where $x_{1}=x \in H, T$ is a nonexpansive mapping of $C$ into $H$ and $F$ is a bifunction from $C \times C$ into $\mathbb{R}$. They prove that, under the sequences $\left\{\alpha_{n}\right\} \subset[\alpha, 1]$ for some $\alpha \in(0,1)$ and $\left\{r_{n}\right\} \subset[r, \infty)$ for some $r>0$, if $\Omega=F(T) \cap \mathrm{EP}(F) \neq \emptyset$, then the sequence $\left\{x_{n}\right\}$ generated by (1.13) converges strongly to $P_{\Omega}\left(x_{1}\right)$ such that $\mathrm{EP}(F)$ is the set of solutions of equilibrium problem defined by

$$
\operatorname{EP}(F)=\{x \in C: F(x, y) \geq 0, \forall y \in C\}
$$

In this paper, we introduce an iterative scheme (1.15) for finding a common element of the set of common fixed points for an infinite family of strictly pseudocontractive mappings and the set of solutions of a system of cocoercive quasivariational inclusions problems by the shrinking projection method in Hilbert spaces as follows:

$$
\begin{gathered}
y_{n}=\alpha_{n} W_{n} x_{n}+\left(1-\alpha_{n}\right) \sum_{i=1}^{N} \beta_{i} J_{M_{i}, \lambda_{i}}\left(x_{n}-\lambda_{i} B_{i} x_{n}\right), \\
\epsilon_{n}=\alpha_{n}\left(1-\alpha_{n}\right)\left\|W_{n} x_{n}-\sum_{i=1}^{N} \beta_{i} J_{M_{i}, \lambda_{i}}\left(x_{n}-\lambda_{i} B_{i} x_{n}\right)\right\|^{2}, \\
C_{n+1}=\left\{z \in C_{n} \cap Q_{n}:\left\|y_{n}-z\right\|^{2} \leq\left\|x_{n}-z\right\|^{2}-\epsilon_{n}\right\}, \\
Q_{n+1}=\left\{z \in C_{n} \cap Q_{n}:\left\langle x_{n}-z, x_{1}-x_{n}\right\rangle \geq 0\right\}, \\
C_{1}=Q_{1}=H, \\
x_{n+1}=P_{C_{n+1} \cap Q_{n+1}}\left(x_{1}\right), \quad \forall n \in \mathbb{N},
\end{gathered}
$$

where $x_{1}=u \in H$ chosen arbitrarily, $M_{i}: H \rightarrow 2^{H}$ is a maximal monotone mapping, $B_{i}: H \rightarrow H$ is a $\xi_{i}$-cocoercive mapping for each $i=1,2, \ldots, N$, and $W_{n}$ is a $W$-mapping on $H$ generated by $\left\{S_{n}\right\}$ and $\left\{\mu_{n}\right\}$ such that the mapping $S_{n}: H \rightarrow H$ defined by $S_{n} x=$ $\alpha x+(1-\alpha) T_{n} x$ for all $x \in H$, where $\left\{T_{n}: H \rightarrow H\right\}$ is an infinite family of $k$-strictly pseudocontractive mappings with a fixed point.

It is well known that the class of strictly pseudocontractive mappings contains the class of nonexpansive mappings, and it follows that, if $k=0$, then the iterative scheme (1.15) is 
reduced to find a common element of the set of common fixed points for an infinite family of nonexpansive mappings and the set of solutions of a system of cocoercive quasivariational inclusions problems in Hilbert spaces.

Furthermore, if $M_{i} \equiv B_{i} \equiv 0$ for all $i=1,2, \ldots, N$ and $\sum_{i=1}^{N} \beta_{i}=1$, then the iterative scheme (1.15) is reduced to extend and improve the results of Kikkawa and Takahashi [8] for finding a common fixed point of an infinite family of $k$-strictly pseudocontractive mappings as follows:

$$
\begin{gathered}
x_{1}=u \in C \text { chosen arbitrarily, } \\
y_{n}=\alpha_{n} W_{n} x_{n}+\left(1-\alpha_{n}\right) x_{n}, \\
\epsilon_{n}=\alpha_{n}\left(1-\alpha_{n}\right)\left\|W_{n} x_{n}-x_{n}\right\|^{2}, \\
C_{n+1}=\left\{z \in C_{n} \cap Q_{n}:\left\|y_{n}-z\right\|^{2} \leq\left\|x_{n}-z\right\|^{2}-\epsilon_{n}\right\}, \\
Q_{n+1}=\left\{z \in C_{n} \cap Q_{n}:\left\langle x_{n}-z, x_{1}-x_{n}\right\rangle \geq 0\right\}, \\
C_{1}=Q_{1}=C, \\
x_{n+1}=P_{C_{n+1} \cap Q_{n+1}}\left(x_{1}\right), \quad \forall n \in \mathbb{N},
\end{gathered}
$$

and if $k=\alpha=0$ and setting $T_{1} \equiv T, T_{n} \equiv I$ for all $n=2,3, \ldots$, then the iterative scheme (1.16) is reduced to find a fixed point of a nonexpansive mapping, for which the convergence rate of the iterative scheme is faster than that of the iterative scheme of Su and Qin [9] as follows:

$$
\begin{gathered}
x_{1}=u \in C \text { chosen arbitrarily, } \\
y_{n}=\sigma_{n} T x_{n}+\left(1-\sigma_{n}\right) x_{n}, \\
\delta_{n}=\sigma_{n}\left(1-\sigma_{n}\right)\left\|T x_{n}-x_{n}\right\|^{2}, \\
D_{n+1}=\left\{z \in D_{n} \cap Q_{\mathrm{n}}:\left\|y_{n}-z\right\|^{2} \leq\left\|x_{n}-z\right\|^{2}-\delta_{n}\right\}, \\
Q_{n+1}=\left\{z \in D_{n} \cap Q_{n}:\left\langle x_{n}-z, x_{1}-x_{n}\right\rangle \geq 0\right\}, \\
D_{1}=Q_{1}=C, \\
x_{n+1}=P_{D_{n+1} \cap Q_{n+1}}\left(x_{1}\right), \quad \forall n \in \mathbb{N} .
\end{gathered}
$$

We suggest and analyze the iterative scheme (1.15) under some appropriate conditions imposed on the parameters. The strong convergence theorem for the above two sets is obtained, and the applicability of the results is shown to extend and improve some well-known results existing in the current literature.

\section{Preliminaries}

We collect the following lemmas which will be used in the proof of the main results in the next section. 
Lemma 2.1 (see [11]). Let $H$ be a Hilbert space. For any $x, y \in H$ and $\lambda \in \mathbb{R}$, one has

$$
\|\lambda x+(1-\lambda) y\|^{2}=\lambda\|x\|^{2}+(1-\lambda)\|y\|^{2}-\lambda(1-\lambda)\|x-y\|^{2} .
$$

Lemma 2.2 (see [1]). Let C be a nonempty closed convex subset of a Hilbert space $H$. Then the following inequality holds:

$$
\left\langle x-P_{C} x, P_{C} x-y\right\rangle \geq 0, \quad \forall x \in H, y \in C .
$$

Lemma 2.3 (see [5]). Let $C$ be a nonempty closed convex subset of a Hilbert space $H$, define mapping $W_{n}$ as (1.8), let $S_{i}: C \rightarrow$ C be a family of infinitely nonexpansive mappings with $\bigcap_{i=1}^{\infty} F\left(S_{i}\right) \neq \emptyset$, and let $\left\{\mu_{i}\right\}$ be a sequence such that $0<\mu_{i} \leq \mu<1$, for all $i \geq 1$. Then

(1) $W_{n}$ is nonexpansive and $F\left(W_{n}\right)=\bigcap_{i=1}^{n} F\left(S_{i}\right)$ for each $n \geq 1$,

(2) for each $x \in C$ and for each positive integer $k, \lim _{n \rightarrow \infty} U_{n, k} x$ exists,

(3) the mapping $W: C \rightarrow C$ defined by

$$
W x:=\lim _{n \rightarrow \infty} W_{n} x=\lim _{n \rightarrow \infty} U_{n, 1} x, \quad x \in C,
$$

is a nonexpansive mapping satisfying $F(W)=\bigcap_{i=1}^{\infty} F\left(S_{i}\right)$ and it is called the $W$-mapping generated by $S_{1}, S_{2}, \ldots$ and $\mu_{1}, \mu_{2}, \ldots$

Lemma 2.4 (see [6]). The resolvent operator $J_{M, \lambda}$ associated with $M$ is single valued and nonexpansive for all $\lambda>0$.

Lemma 2.5 (see [6]). $u \in H$ is a solution of quasivariational inclusion (1.1) if and only if $u=$ $J_{M, \lambda}(u-\lambda B u)$, for all $\lambda>0$, that is,

$$
\mathrm{VI}(H, B, M)=F\left(J_{M, \lambda}(I-\lambda B)\right), \quad \forall \lambda>0 .
$$

Lemma 2.6 (see [12]). Let $C$ be a nonempty closed convex subset of a strictly convex Banach space $X$. Let $\left\{T_{n}: n \in \mathbb{N}\right\}$ be a sequence of nonexpansive mappings on $C$. Suppose that $\bigcap_{n=1}^{\infty} F\left(T_{n}\right) \neq \emptyset$. Let $\left\{\alpha_{n}\right\}$ be a sequence of positive real numbers such that $\sum_{n=1}^{\infty} \alpha_{n}=1$. Then a mapping $S$ on $C$ defined by

$$
S x=\sum_{n=1}^{\infty} \alpha_{n} T_{n} x
$$

for $x \in C$, is well defined, nonexpansive and $F(S)=\bigcap_{n=1}^{\infty} F\left(T_{n}\right)$ holds.

Lemma 2.7 (see [13]). Let $C$ be a nonempty closed convex subset of a Hilbert space $H$ and $S: C \rightarrow$ $C$ a nonexpansive mapping. Then $I-S$ is demiclosed at zero. That is, whenever $\left\{x_{n}\right\}$ is a sequence in $C$ weakly converging to some $x \in C$ and the sequence $\left\{(I-S) x_{n}\right\}$ strongly converges to some $y$, it follows that $(I-S) x=y$. 
Lemma 2.8 (see [14]). Let $C$ be a nonempty closed convex subset of a real Hilbert space $H$ and $T: C \rightarrow C$ a k-strict pseudocontraction. Define $S: C \rightarrow C$ by $S x=\alpha x+(1-\alpha)$ Tx for each $x \in C$. Then, as $\alpha \in[k, 1), S$ is nonexpansive such that $F(S)=F(T)$.

Lemma 2.9 (see [1]). Every Hilbert space $H$ has Radon-Riesz property or Kadec-Klee property, that is, for a sequence $\left\{x_{n}\right\} \subset H$ with $x_{n} \rightarrow x$ and $\left\|x_{n}\right\| \rightarrow\|x\|$ then $x_{n} \rightarrow x$.

\section{Main Results}

Theorem 3.1. Let $H$ be a real Hilbert space, $M_{i}: H \rightarrow 2^{H}$ a maximal monotone mapping, and $B_{i}$ : $H \rightarrow H$ a $\xi_{i}$-cocoercive mapping for each $i=1,2, \ldots, N$. Let $\left\{T_{n}: H \rightarrow H\right\}$ be an infinite family of $k$-strictly pseudocontractive mappings with a fixed point such that $k \in[0,1)$. Define a mapping $S_{n}: H \rightarrow H$ by

$$
S_{n} x=\alpha x+(1-\alpha) T_{n} x, \quad \forall x \in H,
$$

for all $n \in \mathbb{N}$, where $\alpha \in[k, 1)$. Let $W_{n}: H \rightarrow H$ be a $W$-mapping generated by $\left\{S_{n}\right\}$ and $\left\{\mu_{n}\right\}$ such that $\left\{\mu_{n}\right\} \subset(0, \mu]$, for some $\mu \in(0,1)$. Assume that $\Omega:=\left(\bigcap_{n=1}^{\infty} F\left(T_{n}\right)\right) \cap\left(\bigcap_{i=1}^{N} \operatorname{VI}\left(H, B_{i}, M_{i}\right)\right) \neq \emptyset$. For $x_{1}=u \in H$ chosen arbitrarily, suppose that $\left\{x_{n}\right\}$ is generated iteratively by

$$
\begin{gathered}
y_{n}=\alpha_{n} W_{n} x_{n}+\left(1-\alpha_{n}\right) \sum_{i=1}^{N} \beta_{i} J_{M_{i}, \lambda_{i}}\left(x_{n}-\lambda_{i} B_{i} x_{n}\right), \\
\epsilon_{n}=\alpha_{n}\left(1-\alpha_{n}\right)\left\|W_{n} x_{n}-\sum_{i=1}^{N} \beta_{i} J_{M_{i}, \lambda_{i}}\left(x_{n}-\lambda_{i} B_{i} x_{n}\right)\right\|^{2}, \\
C_{n+1}=\left\{z \in C_{n} \cap Q_{n}:\left\|y_{n}-z\right\|^{2} \leq\left\|x_{n}-z\right\|^{2}-\epsilon_{n}\right\}, \\
Q_{n+1}=\left\{z \in C_{n} \cap Q_{n}:\left\langle x_{n}-z, x_{1}-x_{n}\right\rangle \geq 0\right\}, \\
C_{1}=Q_{1}=H, \\
x_{n+1}=P_{C_{n+1} \cap Q_{n+1}}\left(x_{1}\right), \quad \forall n \in \mathbb{N},
\end{gathered}
$$

where

$$
\begin{aligned}
& \text { (C1) }\left\{\alpha_{n}\right\} \subset[a, b] \text { such that } 0<a<b<1, \\
& \text { (C2) } \beta_{i} \in(0,1) \text { and } \lambda_{i} \in\left(0,2 \xi_{i}\right] \text { for each } i=1,2, \ldots, N \text {, } \\
& \text { (C3) } \sum_{i=1}^{N} \beta_{i}=1 \text {. }
\end{aligned}
$$

Then, the sequences $\left\{x_{n}\right\}$ and $\left\{y_{n}\right\}$ converge strongly to $w=P_{\Omega}\left(x_{1}\right)$.

Proof. For any $x, y \in H$ and for each $i=1,2, \ldots, N$, by the $\xi_{i}$-cocoercivity of $B_{i}$, we have

$$
\begin{aligned}
\left\|\left(I-\lambda_{i} B_{i}\right) x-\left(I-\lambda_{i} B_{i}\right) y\right\|^{2} & =\left\|(x-y)-\lambda_{i}\left(B_{i} x-B_{i} y\right)\right\|^{2} \\
& =\|x-y\|^{2}-2 \lambda_{i}\left\langle x-y, B_{i} x-B_{i} y\right\rangle
\end{aligned}
$$


ISRN Mathematical Analysis

$$
\begin{aligned}
& +\lambda_{i}^{2}\left\|B_{i} x-B_{i} y\right\|^{2} \\
\leq & \|x-y\|^{2}-\left(2 \xi_{i}-\lambda_{i}\right) \lambda_{i}\left\|B_{i} x-B_{i} y\right\|^{2} \\
\leq & \|x-y\|^{2},
\end{aligned}
$$

which implies that $I-\lambda_{i} B_{i}$ is nonexpansive. Pick $p \in \Omega$. Therefore, by Lemma 2.5, we have

$$
p=J_{M_{i}, \lambda_{i}}\left(I-\lambda_{i} B_{i}\right) p
$$

for each $i=1,2, \ldots, N$. Since $S_{n} x=\alpha x+(1-\alpha) T_{n} x$, where $\alpha \in[k, 1)$ and $\left\{T_{n}\right\}$ is a family of $k$-strict pseudocontraction, therefore, by Lemma 2.8 , we have that $S_{n}$ is nonexpansive and $F\left(S_{n}\right)=F\left(T_{n}\right)$. It follows from Lemma 2.3(1) that $F\left(W_{n}\right)=\bigcap_{i=1}^{n} F\left(S_{i}\right)=\bigcap_{i=1}^{n} F\left(T_{i}\right)$, which implies that $W_{n} p=p$. Therefore, by (C3), (3.4), Lemma 2.1, and the nonexpansiveness of $W_{n}, J_{M_{i}, \lambda_{i}}$, and $I-\lambda_{i} B_{i}$, we have

$$
\begin{aligned}
\left\|y_{n}-p\right\|^{2}= & \left\|\alpha_{n} W_{n} x_{n}+\left(1-\alpha_{n}\right) \sum_{i=1}^{N} \beta_{i} J_{M_{i}, \lambda_{i}}\left(x_{n}-\lambda_{i} B_{i} x_{n}\right)-p\right\|^{2} \\
= & \left\|\alpha_{n}\left(W_{n} x_{n}-p\right)+\left(1-\alpha_{n}\right) \sum_{i=1}^{N} \beta_{i}\left(J_{M_{i}, \lambda_{i}}\left(x_{n}-\lambda_{i} B_{i} x_{n}\right)-p\right)\right\|^{2} \\
= & \alpha_{n}\left\|W_{n} x_{n}-W_{n} p\right\|^{2} \\
& +\left(1-\alpha_{n}\right)\left\|\sum_{i=1}^{N} \beta_{i}\left(J_{M_{i}, \lambda_{i}}\left(x_{n}-\lambda_{i} B_{i} x_{n}\right)-J_{M_{i}, \lambda_{i}}\left(p-\lambda_{i} B_{i} p\right)\right)\right\|^{2} \\
& -\alpha_{n}\left(1-\alpha_{n}\right)\left\|W_{n} x_{n}-\sum_{i=1}^{N} \beta_{i} J_{M_{i}, \lambda_{i}}\left(x_{n}-\lambda_{i} B_{i} x_{n}\right)\right\|^{2} \\
\leq & \alpha_{n}\left\|x_{n}-p\right\|^{2} \\
& +\left(1-\alpha_{n}\right)\left(\sum_{i=1}^{N} \beta_{i}\left\|\left(x_{n}-\lambda_{i} B_{i} x_{n}\right)-\left(p-\lambda_{i} B_{i} p\right)\right\|\right)^{2}-\epsilon_{n} \\
\leq & \alpha_{n}\left\|x_{n}-p\right\|^{2}+\left(1-\alpha_{n}\right)\left\|x_{n}-p\right\|^{2}-\epsilon_{n} \\
= & \left\|x_{n}-p\right\|^{2}-\epsilon_{n},
\end{aligned}
$$

for all $n \in \mathbb{N}$. Firstly, we prove that $C_{n} \cap Q_{n}$ is closed and convex for all $n \in \mathbb{N}$. It is obvious that $C_{1} \cap Q_{1}$ is closed and, by mathematical induction, that $C_{n} \cap Q_{n}$ is closed for all $n \geq 2$, that is $C_{n} \cap Q_{n}$ is closed for all $n \in \mathbb{N}$. Since $\left\|y_{n}-z\right\|^{2} \leq\left\|x_{n}-z\right\|^{2}-\epsilon_{n}$ is equivalent to

$$
\left\|y_{n}-x_{n}\right\|^{2}+2\left\langle y_{n}-x_{n}, x_{n}-z\right\rangle+\epsilon_{n} \leq 0
$$


for all $n \in \mathbb{N}$, therefore, for any $z_{1}, z_{2} \in C_{n+1} \cap Q_{n+1} \subset C_{n} \cap Q_{n}$ and $\epsilon \in(0,1)$, we have

$$
\begin{aligned}
\| y_{n}- & x_{n} \|^{2}+2\left\langle y_{n}-x_{n}, x_{n}-\left(\epsilon z_{1}+(1-\epsilon) z_{2}\right)\right\rangle+\epsilon_{n} \\
= & \epsilon\left(\left\|y_{n}-x_{n}\right\|^{2}+2\left\langle y_{n}-x_{n}, x_{n}-z_{1}\right\rangle+\epsilon_{n}\right) \\
& +(1-\epsilon)\left(\left\|y_{n}-x_{n}\right\|^{2}+2\left\langle y_{n}-x_{n}, x_{n}-z_{2}\right\rangle+\epsilon_{n}\right) \\
\leq & 0,
\end{aligned}
$$

for all $n \in \mathbb{N}$, and we have

$$
\left\langle x_{n}-\left(\epsilon z_{1}+(1-\epsilon) z_{2}\right), x_{1}-x_{n}\right\rangle=\epsilon\left\langle x_{n}-z_{1}, x_{1}-x_{n}\right\rangle+(1-\epsilon)\left\langle x_{n}-z_{2}, x_{1}-x_{n}\right\rangle \geq 0,
$$

for all $n \in \mathbb{N}$. Since $C_{1} \cap Q_{1}$ is convex, and by putting $n=1$ in (3.6), (3.7), and (3.8), we have that $C_{2} \cap Q_{2}$ is convex. Suppose that $x_{k}$ is given and $C_{k} \cap Q_{k}$ is convex for some $k \geq 2$. It follows by putting $n=k$ in (3.6), (3.7), and (3.8) that $C_{k+1} \cap Q_{k+1}$ is convex. Therefore, by mathematical induction, we have that $C_{n} \cap Q_{n}$ is convex for all $n \geq 2$, that is, $C_{n} \cap Q_{n}$ is convex for all $n \in \mathbb{N}$. Hence, we obtain that $C_{n} \cap Q_{n}$ is closed and convex for all $n \in \mathbb{N}$.

Next, we prove that $\Omega \subset C_{n} \cap Q_{n}$ for all $n \in \mathbb{N}$. It is obvious that $p \in \Omega \subset H=C_{1} \cap Q_{1}$. Therefore, by (3.2) and (3.5), we have $p \in C_{2}$ and note that $p \in H=Q_{2}$, and so $p \in C_{2} \cap Q_{2}$. Hence, we have $\Omega \subset C_{2} \cap Q_{2}$. Since $C_{2} \cap Q_{2}$ is a nonempty closed convex subset of $H$, there exists a unique element $x_{2} \in C_{2} \cap Q_{2}$ such that $x_{2}=P_{C_{2} \cap Q_{2}}\left(x_{1}\right)$. Suppose that $x_{k} \in C_{k} \cap Q_{k}$ is given such that $x_{k}=P_{C_{k} \cap Q_{k}}\left(x_{1}\right)$, and $p \in \Omega \subset C_{k} \cap Q_{k}$ for some $k \geq 2$. Therefore, by (3.2) and (3.5), we have $p \in C_{k+1}$. Since $x_{k}=P_{C_{k} \cap Q_{k}}\left(x_{1}\right)$, therefore, by Lemma 2.2, we have

$$
\left\langle x_{k}-z, x_{1}-x_{k}\right\rangle \geq 0
$$

for all $z \in C_{k} \cap Q_{k}$. Thus, by (3.2), we have $p \in Q_{k+1}$, and so $p \in C_{k+1} \cap Q_{k+1}$. Hence, we have $\Omega \subset C_{k+1} \cap Q_{k+1}$. Since $C_{k+1} \cap Q_{k+1}$ is a nonempty closed convex subset of $H$, there exists a unique element $x_{k+1} \in C_{k+1} \cap Q_{k+1}$ such that $x_{k+1}=P_{C_{k+1} \cap Q_{k+1}}\left(x_{1}\right)$. Therefore, by mathematical induction, we obtain $\Omega \subset C_{n} \cap Q_{n}$ for all $n \geq 2$, and so $\Omega \subset C_{n} \cap Q_{n}$ for all $n \in \mathbb{N}$, and we can define $x_{n+1}=P_{C_{n+1} \cap Q_{n+1}}\left(x_{1}\right)$ for all $n \in \mathbb{N}$. Hence, we obtain that the iteration (3.2) is well defined.

Next, we prove that $\left\{x_{n}\right\}$ is bounded. Since $x_{n}=P_{C_{n} \cap Q_{n}}\left(x_{1}\right)$ for all $n \in \mathbb{N}$, we have

$$
\left\|x_{n}-x_{1}\right\| \leq\left\|z-x_{1}\right\|
$$

for all $z \in C_{n} \cap Q_{n}$. It follows by $p \in \Omega \subset C_{n} \cap Q_{n}$ that $\left\|x_{n}-x_{1}\right\| \leq\left\|p-x_{1}\right\|$ for all $n \in \mathbb{N}$. This implies that $\left\{x_{n}\right\}$ is bounded, and so is $\left\{y_{n}\right\}$.

Next, we prove that $\left\|y_{n}-x_{n}\right\| \rightarrow 0$ as $n \rightarrow \infty$. Since $x_{n+1}=P_{C_{n+1} \cap Q_{n+1}}\left(x_{1}\right) \in C_{n+1} \cap$ $Q_{n+1} \subset C_{n} \cap Q_{n}$, therefore, by (3.10), we have $\left\|x_{n}-x_{1}\right\| \leq\left\|x_{n+1}-x_{1}\right\|$ for all $n \in \mathbb{N}$. This implies that $\left\{\left\|x_{n}-x_{1}\right\|\right\}$ is a bounded nondecreasing sequence and there exists the limit of $\left\|x_{n}-x_{1}\right\|$, that is,

$$
\lim _{n \rightarrow \infty}\left\|x_{n}-x_{1}\right\|=m,
$$


for some $m \geq 0$. Since $x_{n+1} \in Q_{n+1}$, therefore, by (3.2), we have

$$
\left\langle x_{n}-x_{n+1}, x_{1}-x_{n}\right\rangle \geq 0 .
$$

It follows by (3.12) that

$$
\begin{aligned}
\left\|x_{n}-x_{n+1}\right\|^{2}= & \left\|\left(x_{n}-x_{1}\right)+\left(x_{1}-x_{n+1}\right)\right\|^{2} \\
= & \left\|x_{n}-x_{1}\right\|^{2}+2\left\langle x_{n}-x_{1}, x_{1}-x_{n}\right\rangle \\
& +2\left\langle x_{n}-x_{1}, x_{n}-x_{n+1}\right\rangle+\left\|x_{n+1}-x_{1}\right\|^{2} \\
\leq & \left\|x_{n+1}-x_{1}\right\|^{2}-\left\|x_{n}-x_{1}\right\|^{2} .
\end{aligned}
$$

Therefore, by (3.11), we have

$$
\left\|x_{n}-x_{n+1}\right\| \longrightarrow 0 \text { as } n \longrightarrow \infty
$$

Since $x_{n+1} \in C_{n+1}$, therefore, by (3.2), we have

$$
\begin{aligned}
\left\|y_{n}-x_{n+1}\right\|^{2} & \leq\left\|x_{n}-x_{n+1}\right\|^{2}-\epsilon_{n} \\
& \leq\left\|x_{n}-x_{n+1}\right\|^{2} .
\end{aligned}
$$

It follows by (3.15) that

$$
\begin{aligned}
\left\|y_{n}-x_{n}\right\| & \leq\left\|y_{n}-x_{n+1}\right\|+\left\|x_{n+1}-x_{n}\right\| \\
& \leq\left\|x_{n}-x_{n+1}\right\|+\left\|x_{n+1}-x_{n}\right\| \\
& =2\left\|x_{n}-x_{n+1}\right\| .
\end{aligned}
$$

Therefore, by (3.14), we obtain

$$
\left\|y_{n}-x_{n}\right\| \longrightarrow 0 \text { as } n \longrightarrow \infty
$$

Since $\left\{x_{n}\right\}$ is bounded, there exists a subsequence $\left\{x_{n_{i}}\right\}$ of $\left\{x_{n}\right\}$ which converges weakly to $\bar{w}$. Next, we prove that $\bar{w} \in \Omega$. Define the sequence of mappings $\left\{Q_{n}: H \rightarrow H\right\}$ and the mapping $Q: H \rightarrow H$ by

$$
\begin{gathered}
Q_{n} x=\alpha_{n} W_{n} x+\left(1-\alpha_{n}\right) \sum_{i=1}^{N} \beta_{i} J_{M_{i}, \lambda_{i}}\left(I-\lambda_{i} B_{i}\right) x, \quad \forall x \in H, \\
Q x=\lim _{n \rightarrow \infty} Q_{n} x,
\end{gathered}
$$

for all $n \in \mathbb{N}$. Therefore, by (C1) and Lemma 2.3(3), we have 


$$
Q x=c W x+(1-c) \sum_{i=1}^{N} \beta_{i} J_{M_{i}, \lambda_{i}}\left(I-\lambda_{i} B_{i}\right) x, \quad \forall x \in H,
$$

where $a \leq c=\lim _{n \rightarrow \infty} \alpha_{n} \leq b$. From (C3) and Lemma 2.3(3), we have that $W$ and $\sum_{i=1}^{N} \beta_{i} J_{M_{i}, \lambda_{i}}\left(I-\lambda_{i} B_{i}\right)$ are nonexpansive. Therefore, by (C2), (C3), Lemmas 2.3(3), 2.5, 2.6, and 2.8, we have

$$
\begin{aligned}
F(Q) & =F(W) \cap F\left(\sum_{i=1}^{N} \beta_{i} J_{M_{i}, \lambda_{i}}\left(I-\lambda_{i} B_{i}\right)\right) \\
& =\left(\bigcap_{i=1}^{\infty} F\left(S_{i}\right)\right) \cap\left(\bigcap_{i=1}^{N} F\left(J_{M_{i}, \lambda_{i}}\left(I-\lambda_{i} B_{i}\right)\right)\right) \\
& =\left(\bigcap_{i=1}^{\infty} F\left(T_{i}\right)\right) \cap\left(\bigcap_{i=1}^{N} \operatorname{VI}\left(H, B_{i}, M_{i}\right)\right),
\end{aligned}
$$

that is, $F(Q)=\Omega$. From (3.17), we have $\left\|y_{n_{i}}-x_{n_{i}}\right\| \rightarrow 0$ as $i \rightarrow \infty$. Thus, from (3.2) and (3.18), we get $\left\|Q x_{n_{i}}-x_{n_{i}}\right\| \rightarrow 0$ as $i \rightarrow \infty$. It follows from $x_{n_{i}} \rightarrow \bar{w}$ and Lemma 2.7 that $\bar{w} \in F(Q)$, that is, $\bar{w} \in \Omega$.

Since $\Omega$ is a nonempty closed convex subset of $H$, there exists a unique $w \in \Omega$ such that $w=P_{\Omega}\left(x_{1}\right)$. Next, we prove that $x_{n} \rightarrow w$ as $n \rightarrow \infty$. Since $w=P_{\Omega}\left(x_{1}\right)$, we have $\left\|x_{1}-w\right\| \leq\left\|x_{1}-z\right\|$ for all $z \in \Omega$, and it follows that

$$
\left\|x_{1}-w\right\| \leq\left\|x_{1}-\bar{w}\right\|
$$

Since $w \in \Omega \subset C_{n} \cap Q_{n}$, therefore, by (3.10), we have

$$
\left\|x_{1}-x_{n}\right\| \leq\left\|x_{1}-w\right\|
$$

Therefore, by (3.21), (3.22), and the weak lower semicontinuity of norm, we have

$$
\begin{aligned}
\left\|x_{1}-w\right\| & \leq\left\|x_{1}-\bar{w}\right\| \\
& \leq \liminf _{i \rightarrow \infty}\left\|x_{1}-x_{n_{i}}\right\| \\
& \leq \limsup _{i \rightarrow \infty}\left\|x_{1}-x_{n_{i}}\right\| \\
& \leq\left\|x_{1}-w\right\| .
\end{aligned}
$$

It follows that

$$
\left\|x_{1}-w\right\|=\lim _{i \rightarrow \infty}\left\|x_{1}-x_{n_{i}}\right\|=\left\|x_{1}-\bar{w}\right\|
$$

Since $x_{n_{i}} \rightarrow \bar{w}$ as $i \rightarrow \infty$, therefore, we have

$$
\left(x_{1}-x_{n_{i}}\right) \rightarrow\left(x_{1}-\bar{w}\right) \quad \text { as } i \longrightarrow \infty .
$$


Hence, from (3.24), (3.25), the Kadec-Klee property, and the uniqueness of $w=P_{\Omega}\left(x_{1}\right)$, we obtain

$$
x_{n_{i}} \longrightarrow \bar{w}=w \quad \text { as } i \longrightarrow \infty .
$$

It follows that $\left\{x_{n}\right\}$ converges strongly to $w$, and so is $\left\{y_{n}\right\}$. This completes the proof.

Remark 3.2. The iteration (3.2) is the difference with some well known results as the following.

(1) The sequence $\left\{x_{n}\right\}$ is the projection sequence of $x_{1}$ onto $C_{n} \cap Q_{n}$ for all $n \in \mathbb{N}$ such that

$$
C_{1} \cap Q_{1} \supset C_{2} \cap Q_{2} \supset \cdots \supset C_{n} \cap Q_{n} \supset \cdots \supset \Omega
$$

(2) The proof of $\bar{w} \in \Omega$ is simple by the demiclosedness principle because the sequence $\left\{y_{n}\right\}$ is a linear nonexpansive mapping form of the mappings $W_{n}$ and $J_{M_{i}, \lambda_{i}}\left(I-\lambda_{i} B_{i}\right)$.

(3) Solving a common fixed point for an infinite family of strictly pseudocontractive mappings and a system of cocoercive quasivariational inclusions problems by iteration is obtained.

\section{Applications}

Theorem 4.1. Let $H$ be a real Hilbert space, $M_{i}: H \rightarrow 2^{H}$ a maximal monotone mapping, and $B_{i}$ : $H \rightarrow H$ a $\xi_{i}$-cocoercive mapping for each $i=1,2, \ldots, N$. Let $\left\{T_{n}: H \rightarrow H\right\}$ be an infinite family of nonexpansive mappings. Define a mapping $S_{n}: H \rightarrow H$ by

$$
S_{n} x=\alpha x+(1-\alpha) T_{n} x, \quad \forall x \in H,
$$

for all $n \in \mathbb{N}$, where $\alpha \in[0,1)$. Let $W_{n}: H \rightarrow H$ be a $W$-mapping generated by $\left\{S_{n}\right\}$ and $\left\{\mu_{n}\right\}$ such that $\left\{\mu_{n}\right\} \subset(0, \mu]$, for some $\mu \in(0,1)$. Assume that $\Omega:=\left(\bigcap_{n=1}^{\infty} F\left(T_{n}\right)\right) \cap\left(\bigcap_{i=1}^{N} \operatorname{VI}\left(H, B_{i}, M_{i}\right)\right) \neq \emptyset$. For $x_{1}=u \in H$ chosen arbitrarily, suppose that $\left\{x_{n}\right\}$ is generated iteratively by

$$
\begin{gathered}
y_{n}=\alpha_{n} W_{n} x_{n}+\left(1-\alpha_{n}\right) \sum_{i=1}^{N} \beta_{i} J_{M_{i}, \lambda_{i}}\left(x_{n}-\lambda_{i} B_{i} x_{n}\right), \\
\epsilon_{n}=\alpha_{n}\left(1-\alpha_{n}\right)\left\|W_{n} x_{n}-\sum_{i=1}^{N} \beta_{i} J_{M_{i}, \lambda_{i}}\left(x_{n}-\lambda_{i} B_{i} x_{n}\right)\right\|^{2}, \\
C_{n+1}=\left\{z \in C_{n} \cap Q_{n}:\left\|y_{n}-z\right\|^{2} \leq\left\|x_{n}-z\right\|^{2}-\epsilon_{n}\right\}, \\
Q_{n+1}=\left\{z \in C_{n} \cap Q_{n}:\left\langle x_{n}-z, x_{1}-x_{n}\right\rangle \geq 0\right\}, \\
C_{1}=Q_{1}=H, \\
x_{n+1}=P_{C_{n+1} \cap Q_{n+1}}\left(x_{1}\right), \quad \forall n \in \mathbb{N},
\end{gathered}
$$


where

(C1) $\left\{\alpha_{n}\right\} \subset[a, b]$ such that $0<a<b<1$,

(C2) $\beta_{i} \in(0,1)$ and $\lambda_{i} \in\left(0,2 \xi_{i}\right]$ for each $i=1,2, \ldots, N$,

(C3) $\sum_{i=1}^{N} \beta_{i}=1$.

Then the sequences $\left\{x_{n}\right\}$ and $\left\{y_{n}\right\}$ converge strongly to $w=P_{\Omega}\left(x_{1}\right)$.

Proof. It is concluded from Theorem 3.1 immediately, by putting $k=0$.

Theorem 4.2. Let $C$ be a nonempty closed convex subset of a real Hilbert space $H$ and $\left\{T_{n}: C \rightarrow C\right\}$ an infinite family of $k$-strictly pseudocontractive mappings with a fixed point such that $k \in[0,1)$. Define a mapping $S_{n}: C \rightarrow C$ by

$$
S_{n} x=\alpha x+(1-\alpha) T_{n} x, \quad \forall x \in C,
$$

for all $n \in \mathbb{N}$, where $\alpha \in[k, 1)$. Let $W_{n}: C \rightarrow C$ be a $W$-mapping generated by $\left\{S_{n}\right\}$ and $\left\{\mu_{n}\right\}$ such that $\left\{\mu_{n}\right\} \subset(0, \mu]$, for some $\mu \in(0,1)$. Assume that $\Omega:=\bigcap_{n=1}^{\infty} F\left(T_{n}\right) \neq \emptyset$. For $x_{1}=u \in C$ chosen arbitrarily, suppose that $\left\{x_{n}\right\}$ is generated iteratively by

$$
\begin{gathered}
y_{n}=\alpha_{n} W_{n} x_{n}+\left(1-\alpha_{n}\right) x_{n}, \\
\epsilon_{n}=\alpha_{n}\left(1-\alpha_{n}\right)\left\|W_{n} x_{n}-x_{n}\right\|^{2}, \\
C_{n+1}=\left\{z \in C_{n} \cap Q_{n}:\left\|y_{n}-z\right\|^{2} \leq\left\|x_{n}-z\right\|^{2}-\epsilon_{n}\right\}, \\
Q_{n+1}=\left\{z \in C_{n} \cap Q_{n}:\left\langle x_{n}-z, x_{1}-x_{n}\right\rangle \geq 0\right\}, \\
C_{1}=Q_{1}=C, \\
x_{n+1}=P_{C_{n+1} \cap Q_{n+1}}\left(x_{1}\right), \quad \forall n \in \mathbb{N},
\end{gathered}
$$

where $\left\{\alpha_{n}\right\} \subset[a, b]$ such that $0<a<b<1$. Then the sequences $\left\{x_{n}\right\}$ and $\left\{y_{n}\right\}$ converge strongly to $w=P_{\Omega}\left(x_{1}\right)$.

Proof. It is concluded from Theorem 3.1 immediately, by putting $M_{i} \equiv B_{i} \equiv 0$ for all $i=$ $1,2, \ldots, N$.

Theorem 4.3. Let $C$ be a nonempty closed convex subset of a real Hilbert space $H$ and $T: C \rightarrow C$ a nonexpansive mapping. Assume that $F(T) \neq \emptyset$. For $x_{1}=u \in C$ chosen arbitrarily, suppose that $\left\{x_{n}\right\}$ is generated iteratively by

$$
\begin{gathered}
y_{n}=\sigma_{n} T x_{n}+\left(1-\sigma_{n}\right) x_{n} \\
\delta_{n}=\sigma_{n}\left(1-\sigma_{n}\right)\left\|T x_{n}-x_{n}\right\|^{2}, \\
D_{n+1}=\left\{z \in D_{n} \cap Q_{n}:\left\|y_{n}-z\right\|^{2} \leq\left\|x_{n}-z\right\|^{2}-\delta_{n}\right\}, \\
Q_{n+1}=\left\{z \in D_{n} \cap Q_{n}:\left\langle x_{n}-z, x_{1}-x_{n}\right\rangle \geq 0\right\},
\end{gathered}
$$




$$
\begin{gathered}
D_{1}=Q_{1}=C, \\
x_{n+1}=P_{D_{n+1} \cap Q_{n+1}}\left(x_{1}\right), \quad \forall n \in \mathbb{N},
\end{gathered}
$$

where $\left\{\sigma_{n}\right\} \subset[a, b]$ such that $0<a<b<1$. Then the sequences $\left\{x_{n}\right\}$ and $\left\{y_{n}\right\}$ converge strongly to $w=P_{F(T)}\left(x_{1}\right)$.

Proof. It is concluded from Theorem 4.2, by putting $\alpha=0$. Setting $T_{1} \equiv T, T_{n} \equiv I$ for all $n=2,3, \ldots$ and leting $\mu_{n} \subset(0, \mu]$ for some $\mu \in(0,1)$, therefore, from the definition of $S_{n}$ in Theorem 4.2, we have $S_{1}=T_{1}=T$ and $S_{n}=I$ for all $n=2,3, \ldots$ Since $W_{n}$ is a $W$-mapping generated by $\left\{S_{n}\right\}$ and $\left\{\mu_{n}\right\}$, therefore, by the definition of $U_{n, i}$ and $W_{n}$ in (1.8), we have $U_{n, i}=I$ for all $i=2,3, \ldots$ and $W_{n}=U_{n, 1}=\mu_{1} S_{1} U_{n, 2}+\left(1-\mu_{1}\right) I=\mu_{1} T+\left(1-\mu_{1}\right) I$. Hence, by Theorem 4.2, we obtain

$$
\begin{aligned}
y_{n} & =\alpha_{n} W_{n} x_{n}+\left(1-\alpha_{n}\right) x_{n} \\
& =\alpha_{n}\left(\mu_{1} T x_{n}+\left(1-\mu_{1}\right) x_{n}\right)+\left(1-\alpha_{n}\right) x_{n} \\
& =\sigma_{n} T x_{n}+\left(1-\sigma_{n}\right) x_{n}
\end{aligned}
$$

where $\sigma_{n}:=\alpha_{n} \mu_{1}$. Since, the same as in the proof of Theorem 3.1, we have that $D_{n} \cap Q_{n}$ is a nonempty closed convex subset of $C$ for all $n \in \mathbb{N}$ and by Theorem 4.2, we have

$$
\begin{aligned}
\epsilon_{n} & =\alpha_{n}\left(1-\alpha_{n}\right)\left\|W_{n} x_{n}-x_{n}\right\|^{2} \\
& =\alpha_{n}\left(1-\alpha_{n}\right)\left\|\mu_{1} T x_{n}+\left(1-\mu_{1}\right) x_{n}-x_{n}\right\|^{2} \\
& =\left(\alpha_{n} \mu_{1}\right)\left(\mu_{1}-\mu_{1} \alpha_{n}\right)\left\|T x_{n}-x_{n}\right\|^{2} \\
& =\sigma_{n}\left(\mu_{1}-\sigma_{n}\right)\left\|T x_{n}-x_{n}\right\|^{2} \\
& \leq \sigma_{n}\left(1-\sigma_{n}\right)\left\|T x_{n}-x_{n}\right\|^{2} \\
& =\delta_{n}
\end{aligned}
$$

for all $n \in \mathbb{N}$. It follows that $D_{n} \subset C_{n}$ for all $n \in \mathbb{N}$, where $C_{n}$ is defined as in Theorem 4.2. Hence, by Theorem 4.2, we obtain the desired result. This completes the proof.

\section{References}

[1] W. Takahashi, Nonlinear Functional Analysis, Fixed Point Theory and its Application, Yokohama Publishers, Yokohama, Japan, 2000.

[2] K. Aoyama, Y. Kimura, W. Takahashi, and M. Toyoda, "Approximation of common fixed points of a countable family of nonexpansive mappings in a Banach space," Nonlinear Analysis. Theory, Methods $\mathcal{E}$ Applications Series A, vol. 67, no. 8, pp. 2350-2360, 2007.

[3] H. H. Bauschke, "The approximation of fixed points of compositions of nonexpansive mappings in Hilbert space," Journal of Mathematical Analysis and Applications, vol. 202, no. 1, pp. 150-159, 1996.

[4] M. Shang, Y. Su, and X. Qin, "Strong convergence theorems for a finite family of nonexpansive mappings," Fixed Point Theory and Applications, vol. 2007, Article ID 76971, 9 pages, 2007. 
[5] K. Shimoji and W. Takahashi, "Strong convergence to common fixed points of infinite nonexpansive mappings and applications," Taiwanese Journal of Mathematics, vol. 5, no. 2, pp. 387-404, 2001.

[6] S.-S. Zhang, J. H. W. Lee, and C. K. Chan, "Algorithms of common solutions to quasi variational inclusion and fixed point problems," Applied Mathematics and Mechanics, vol. 29, no. 5, pp. 571-581, 2008.

[7] K. Nakajo and W. Takahashi, "Strong convergence theorems for nonexpansive mappings and nonexpansive semigroups," Journal of Mathematical Analysis and Applications, vol. 279, no. 2, pp. 372-379, 2003.

[8] M. Kikkawa and W. Takahashi, "Approximating fixed points of infinite nonexpansive mappings by the hybrid method," Journal of Optimization Theory and Applications, vol. 117, no. 1, pp. 93-101, 2003.

[9] Y. Su and X. Qin, "Strong convergence of monotone hybrid method for fixed point iteration processes," Journal of Systems Science E Complexity, vol. 21, no. 3, pp. 474-482, 2008.

[10] A. Tada and W. Takahashi, "Weak and strong convergence theorems for a nonexpansive mapping and an equilibrium problem," Journal of Optimization Theory and Applications, vol. 133, no. 3, pp. 359-370, 2007.

[11] W. Takahashi, Introduction to Nonlinear and Convex Analysis, Yokohama Publishers, Yokohama, Japan, 2009.

[12] R. E. Bruck, Jr., "Properties of fixed-point sets of nonexpansive mappings in Banach spaces," Transactions of the American Mathematical Society, vol. 179, pp. 251-262, 1973.

[13] F. E. Browder, "Nonlinear monotone operators and convex sets in Banach spaces," Bulletin of the American Mathematical Society, vol. 71, pp. 780-785, 1965.

[14] F. E. Browder and W. V. Petryshyn, "Construction of fixed points of nonlinear mappings in Hilbert space," Journal of Mathematical Analysis and Applications, vol. 20, pp. 197-228, 1967. 


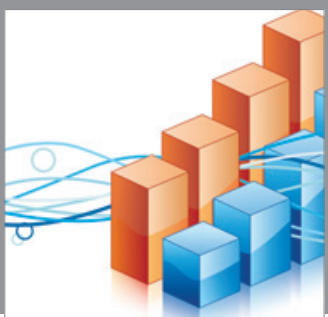

Advances in

Operations Research

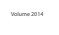

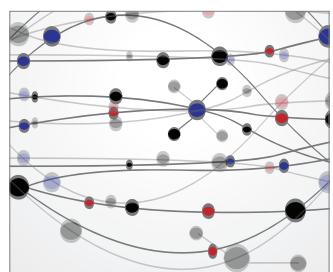

\section{The Scientific} World Journal
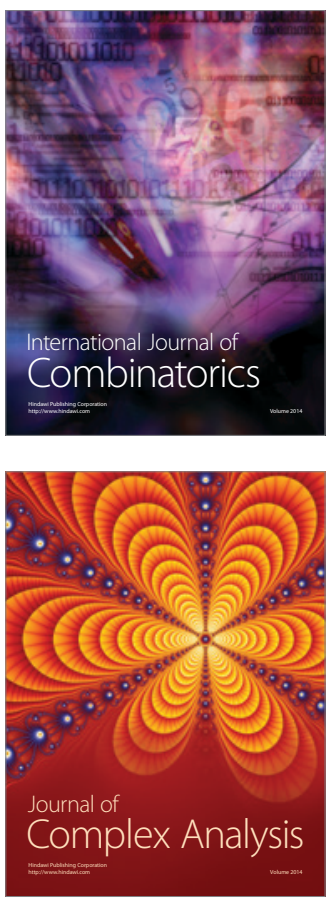

International Journal of

Mathematics and

Mathematical

Sciences
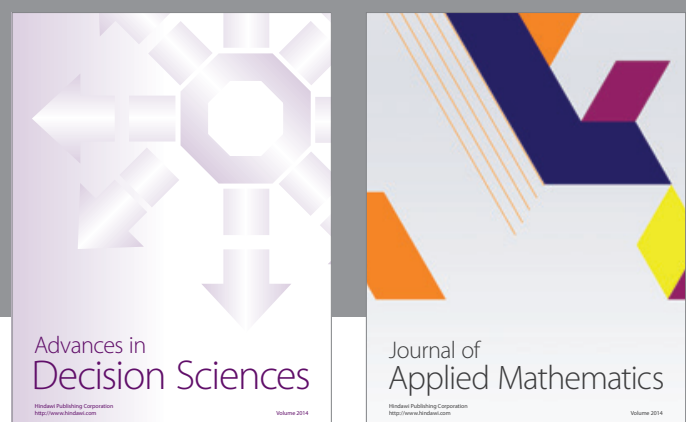

Journal of

Applied Mathematics
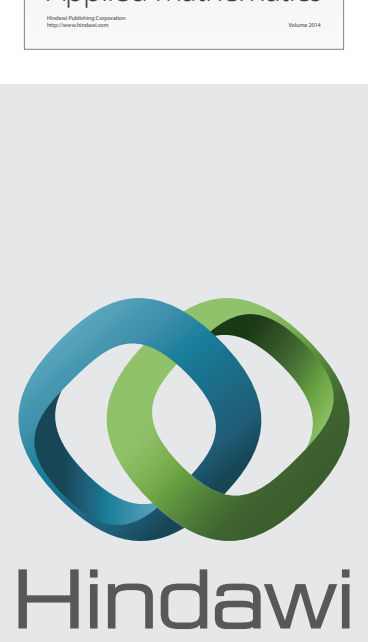

Submit your manuscripts at http://www.hindawi.com
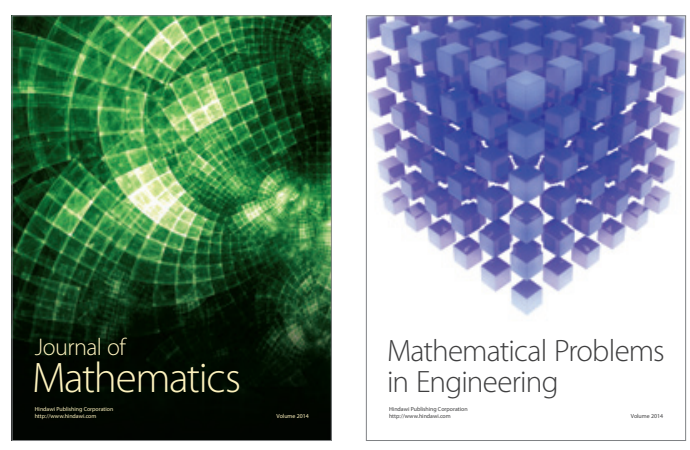

Mathematical Problems in Engineering
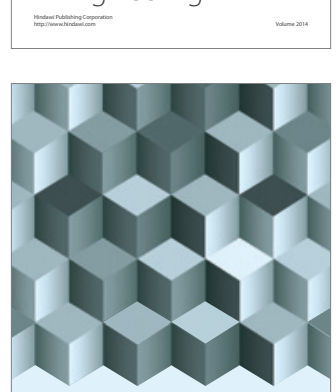

Journal of

Function Spaces
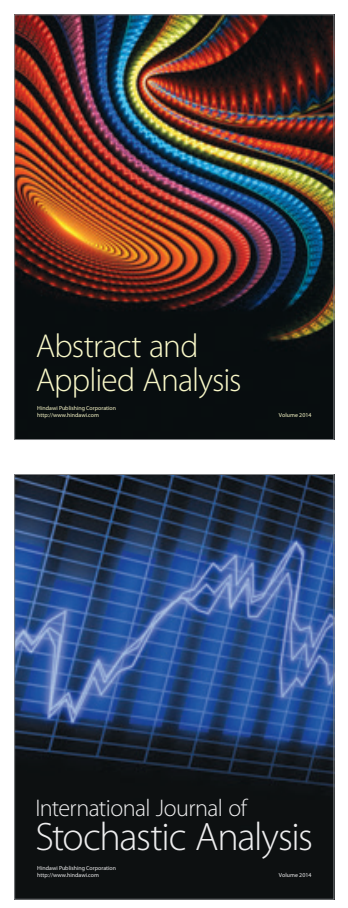

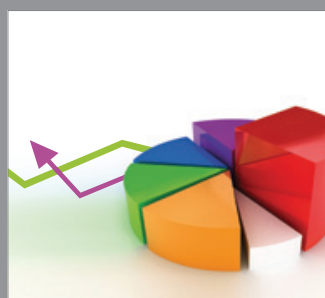

ournal of

Probability and Statistics

Promensencen
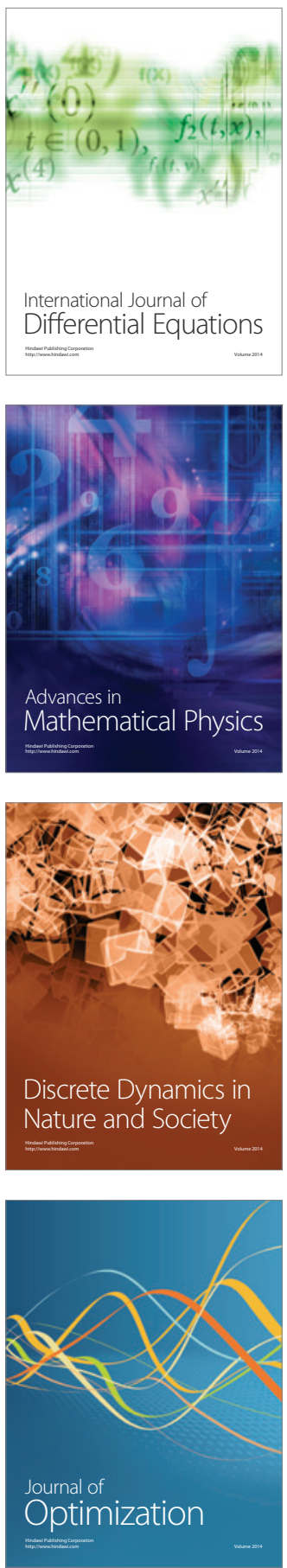